\title{
Yann ALGAN, Elisabeth BEASLEY, Daniel COHEN et Martial FOUCAULT, Les Origines du populisme. Enquête sur un schisme politique et social
}

Martin Deleixhe

\section{(2) OpenEdition}

Journals

Édition électronique

URL : http://journals.openedition.org/ress/6697

DOI : $10.4000 /$ ress.6697

ISBN : 1663-4446

ISSN : $1663-4446$

Éditeur

Librairie Droz

Édition imprimée

Date de publication : 1 décembre 2020

Pagination : 247-250

ISSN : 0048-8046

Référence électronique

Martin Deleixhe, "Yann ALGAN, Elisabeth BEASLEY, Daniel COHEN et Martial FOUCAULT, Les Origines du populisme. Enquête sur un schisme politique et social », Revue européenne des sciences sociales [En ligne], 58-2 | 2020, mis en ligne le 01 décembre 2020, consulté le 02 décembre 2020. URL : http:// journals.openedition.org/ress/6697 ; DOI : https://doi.org/10.4000/ress.6697

Ce document a été généré automatiquement le 2 décembre 2020.

(c) Librairie Droz 


\title{
Yann ALGAN, Elisabeth BEASLEY, Daniel COHEN et Martial FOUCAULT, Les Origines du populisme. Enquête sur un schisme politique et social
}

\author{
Martin Deleixhe
}

\section{RÉFÉRENCE}

Yann ALGAN, Elisabeth BEASLEY, Daniel COHEN et Martial FOUCAULT, 2019, Les Origines du populisme. Enquête sur un schisme politique et social, Paris, Éditions du Seuil, 194 p.

1 Le populisme s'est désormais établi comme un objet central de la science politique, doublé d'un phénomène éditorial majeur. La tentation est par conséquent forte de chercher à monter en marche dans ce train de publications lancé à toute allure, afin de ne pas être en reste et de participer à la conversation scientifique du moment. Cet empressement peut cependant avoir des effets délétères, ainsi que le démontre - à son corps défendant - l'ouvrage co-écrit par Yann Algan, Elisabeth Beasley, Daniel Cohen et Martial Foucault.

2 En lui donnant pour titre Les Origines du populisme, les auteure-s de cette étude (ou fautil y voir une intervention de leur éditeur ?) laissent entendre aux lecteurs et lectrices que l'ouvrage apportera des éclairages sur les causes de ce phénomène. Or, disons-le de suite, il n'y a rien - ou presque - à apprendre au sujet du populisme dans ces pages. Ce qui ne veut pas dire que le livre soit dénué d'intérêt, au contraire. Mais son titre est éminemment trompeur. Car ce que l'ouvrage se propose en réalité de faire, comme le donne à deviner son sous-titre Enquête sur un schisme politique et social, c'est de soumettre à l'examen la thèse selon laquelle deux variables sociologiques préalablement peu exploitées par la sociologie électorale, à savoir le degré de confiance interpersonnelle et le degré de satisfaction subjective de chaque individu, offrent des 
capacités explicatives des nouveaux comportements de vote des électeurs nettement supérieures à des variables sociologiques plus classiques comme la classe, le genre ou le statut socio-professionnel.

3 L'inadéquation du titre est d'ailleurs patente dès l'introduction. Pour expliciter ce que le concept de populisme recouvre à leurs yeux, les auteurees ne disent rien de la vaste littérature désormais disponible sur le sujet et ne considèrent aucune des différentes définitions en circulation. Pour délimiter les contours théoriques de ce qui est présenté comme l'objet premier de leur investigation, ils ne se référent qu'à un seul ouvrage (Les Nouveaux Populismes de Dominique Reynié) dont ils retiennent que le terme de populisme est : « imprécis quand il vise à caractériser [...] l'ensemble des détestations qui s'expriment contre les élites, contre le "système" » (p. 17). Quelques lignes à peine après avoir invité à ne pas confondre le populisme avec le rejet des élites ou la défiance envers les institutions, les auteure's écrivent cependant : "Il [le concept de " populisme »] ne permet pas, en particulier, de distinguer les versants gauche et droite de cette protestation. Pour éviter cette ambiguïté, nous utilisons dans ce livre deux expressions distinctes : la "gauche radicale" et la "droite populiste", deux forces unies dans leur critique du "système" [je souligne], mais qui s'opposent profondément sur le terrain des valeurs et soutiennent des plateformes économiques très différentes. » (ibid.). Le lecteur n'y comprend plus rien. Si le populisme ne s'assimile pas à une attitude anti-système, pourquoi ranger deux courants politiques présentés comme distincts - gauche radicale et droite populiste - sous la bannière du populisme précisément en raison de leur opposition à un "système", d'ailleurs indéfini ? Est-ce que cela ne fait pas du rejet des élites et des institutions politiques établies le critère distinctif du populisme? Le cas échéant, est-ce que l'on n'est pas confronté au genre d'imprécision conceptuelle que les auteure-s dénonçaient quelques lignes plus tôt ?

Un point de terminologie rend la question encore plus saillante. Pourquoi appeler la droite « populiste » alors que la gauche, elle, est plutôt qualifiée de " radicale » ? Estce qu'il n'existe pas de gauche populiste? Ou faut-il en conclure que toute la gauche radicale est forcément populiste? Les auteur·es, qui s'appuient beaucoup sur le cas français, font ainsi de la France insoumise de Jean-Luc Mélenchon l'exemple même de la gauche radicale (et donc implicitement populiste). Mais comment faut-il alors qualifier les autres formations de l'extrême gauche du spectre politique en France? Que dire du PCF, par exemple? Radical, il l'est sans aucun doute, puisque que sa plateforme politique vise à renverser le système économique en place. Est-il populiste pour autant? Nombre d'auteurs spécialistes du populisme distinguent pourtant clairement les partis d'ascendance socialistes, qui se réclament d'une science de la société et de ses dynamiques historiques pour justifier leurs positions politiques, des partis politiques qui ont une vision manichéenne du conflit social, opposant les élites corrompues à un peuple unifié et paré de toutes les vertus morales.

5 Ce manque de rigueur terminologique semble tenir au fait que l'ambition des auteurs n'est pas de contribuer à la conversation en cours entre spécialistes du populisme. La bibliographie de l'ouvrage ne se réfère d'ailleurs aucunement à ce pan de la littérature scientifique. L'apport majeur du livre se situe plutôt dans l'innovation qu'elle suggère d'introduire dans le domaine de la sociologie électorale. Envisagé sous cet angle, l'ouvrage se révèle alors riche et instructif. Ses analyses ne sont pas originales mais elles ont le mérite d'être étayées par de robustes données quantitatives, qui permettent 
de confirmer empiriquement certaines réflexions avancées de façon plus spéculatives dans les champs de la sociologie ou de la théorie politique.

6 L'ouvrage exploite des enquêtes mensuelles réalisées sur la base de questionnaires et menées par le CEVIPOF de Sciences Po entre novembre 2015 et juin 2017 auprès de 24000 électeurs français. Réalisées à partir de questionnaires, ces enquêtes avaient la particularité d'interroger les individus non seulement sur un ensemble de données objectives (niveau de diplôme et de revenus, situation familiale, préférences en matière de politiques publiques) mais également sur des variables subjectives, telles que la perception de leur niveau de bien-être, leur degré de confiance en autrui ou leurs valeurs politiques.

7 Il en ressort que les comportements électoraux se sont individualisés. Ce qui ne veut pas dire que certains déterminants sociologiques lourds comme le degré d'éducation, de revenu ou le statut socio-professionnel ne sont plus des variables explicatives pertinentes $\mathrm{du}$ vote. Les comportements électoraux suivent toujours certaines tendances collectives. Mais cette analyse classique gagne aujourd'hui à être affinée, notamment en prenant en considération le degré de confiance en autrui et la perception du bien-être. Ces données sont, certes, partiellement corrélées avec les déterminants objectifs évoqués plus haut. Il n'est pas très surprenant qu'un cadre supérieur soit plus enclin à se déclarer satisfaits de sa qualité de vie qu'un ouvrier non qualifié, ni qu'un chômeur de longue durée manifeste une défiance généralisée. Mais ces variables subjectives sont aussi « le réceptacle des frustrations et des réussites accumulées tout au long d'une vie»(p.62) Dans une société qui s'est atomisée, les consciences de classe ont cédé du terrain à une appréciation plus hétérogène de la situation politique par des personnes qui partagent pourtant des conditions sociales similaires.

8 Pour explorer l'impact de la confiance et du bien-être subjectif sur les comportements électoraux, les auteur'e's mènent une analyse quantitative détaillée et minutieuse des résultats des élections présidentielles françaises (ch. 2 à 6). Pour tester la robustesse de ces premières conclusions, ils conduisent par la suite une série de comparaisons internationales qui corroborent largement les résultats français (ch. 8). Cette analyse quantitative leur permet de mettre en évidence que l'axe gauche-droite "classique " est loin d'avoir disparu. Dans les réponses aux questions qui portent sur des enjeux économique (du type : faudrait-il, en France, plus, autant ou moins de dépenses publiques pour les aides sociales?) ou sur des enjeux culturels (comme faudrait-il, en France, plus, autant ou moins d'expulsions d'immigrés clandestins?), une grande partie des personnes interrogées donnent des réponses polarisées qui dénotent la persistance de deux camps électoraux, la droite et la gauche.

9 Ce qui mérite cependant de retenir l'attention, c'est que certaines catégories d'électeurs brouillent désormais cette classification, et singulièrement les électorats d'Emmanuel Macron et de Marine Le Pen. Ces électeurs sont relativement indifférents aux questions économiques. Ils n'ont pas d'avis tranché sur le niveau optimal de redistribution économique ou de dépenses sociales. Ils n'ont donc pas de positionnement clair sur le continuum gauche-droite. En revanche, si l'on observe de près la variable «bien-être ", ces deux électorats se regroupent de part et d'autre d'un nouvel axe politique, perpendiculaire à l'axe gauche-droite. D'un côté, on retrouve les mécontents, c'est-à-dire les personnes qui jugent avoir une qualité de vie faible, notamment en raison d'une insécurité économique réelle ou perçue, et qui votent 
massivement pour ce que les auteur·e's désignent comme les partis populistes, c'est à dire Le Pen et Mélenchon en France. De l'autre côté, les enthousiastes, c'est-à-dire ceux qui apprécient leur qualité de vie et indiquent être confiants dans l'avenir, votent très majoritairement pour Macron. L'origine du vote « populiste » serait donc à chercher du côté de cette dégradation, réelle ou perçue, de la qualité de vie par des populations dont les conditions sociales peuvent être variées mais qui se sentent menacées par l'ouverture de la France sur l'Europe et le monde. En somme, cet axe esquisse un clivage nouveau qui sépare les « gagnants » des « perdants » de la mondialisation.

Cependant le vote dit populiste est loin de présenter un front unifié. Au sein même du camp "populiste", un net partage des eaux s'opère entre gauche et droite, prioritairement autour des enjeux culturels. Les électeurs de Mélenchon rejoignent sur ce plan la position des électeurs de la gauche classique et embrassent un certain libéralisme culturel, tandis que ceux de Le Pen se rangent du côté de la droite traditionnelle, qui se reconnaît dans les valeurs d'ordre et d'autorité. De façon intéressante, les enquêtes révèlent que cette distinction est fortement corrélée à la notion de confiance interpersonnelle. Les électeurs de Le Pen se défient de tout et de tout le monde, des très pauvres (les « assistés ») comme des plus riches (les «élites ») signalant une relation dégradée au monde social et à autrui. Pour les électeurs de Mélenchon, la critique des élites ne s'accompagne pas d'une défiance généralisée. Au contraire, le haut degré de confiance interpersonnelle manifesté par l'électorat de Mélenchon résonne avec un programme politique qui met en avant la valeur de la solidarité, notamment avec les étrangers, qui vante l'efficacité de la redistribution économique ou qui prend la défense des services publics et des dépenses sociales.

11 Considérés ensemble, la confiance interpersonnelle et le bien-être subjectif dessinent alors un tableau à quatre quadrants. Les mécontents portent leur choix sur des partis dits populistes, mais partagent leur voix entre gauche radicale et droite populiste en fonction de leur degré de confiance en autrui. En revanche, les électeurs heureux n'ont pas les mêmes griefs à l'égard des élites en place ou des institutions existantes et sont donc plus enclins à voter pour des partis traditionnels. À nouveau, la ventilation des voix entre la droite traditionnelle et la gauche socio-démocrate est fortement corrélée au taux de confiance. Les électeurs de la droite traditionnelle ne manifestent pas la même méfiance généralisée que les électeurs de la droite populiste. Leur confiance tend à se limiter au cercle de leur famille et de leurs proches. C'est à l'encontre des personnes qu'ils ne connaissent peu ou pas qu'ils se montrent méfiants. Les électeurs de la gauche socio-démocrate affichent un plus grand optimisme quant à la nature humaine et se montrent confiants même à l'égard d'inconnus.

12 En résumé, si cet ouvrage ne permet pas vraiment d'en apprendre plus sur le phénomène du populisme, faute d'une définition opératoire (et qu'il est donc desservi par son titre), il a cependant le mérite de renseigner - à grand renfort de données quantitatives - sur les mouvements souterrains qu'ont connus les motivations des électeurs et de mettre en exergue leur lien de causalité avec la montée en puissance de partis non-traditionnels qui ont su tirer profit d'un ressentiment social latent et d'une dégradation de la confiance interpersonnelle. 


\section{AUTEURS}

MARTIN DELEIXHE

Université Paris 1 - Panthéon Sorbonne 